\title{
INTOXICACIÓN PEDIÁTRICA POR PARAQUAT: PRESENTACIÓN DE CASO, HOSPITAL INFANTIL DR. ROBERT REID CABRAL (HIRRC), REPÚBLICA DOMINICANA
}

\author{
Paraquat Poisoning in Children: Case Presentation, Hospital Infantil \\ Dr. Robert Reid Cabral, Dominican Republic
}

\author{
Dra. Cristina Contreras*, Dra. Lisandra Corona**, Dra. Norys Domínguez***, \\ Dr. Miguel Mejía Sang****
}

Fecha de recibido: 3 abril 2018 • Fecha de aprobado: 6 junio 2018

Cómo citar: Contreras C, Corona L, Domínguez N, Mejía Sang M. Intoxicación pediátrica por paraquat: presentación de caso, Hospital Infantil Dr. Robert Reid Cabral (HIRRC), República Dominicana. cysa [Internet]. 11 abr. 2019 [citado 12 abr. 2019];1(1):73-8. Available from: https://revistas.intec.edu.do/index.php/cisa/article/view/1334

\section{Resumen}

Introducción: El paraquat (dicloruro de 1,1'-dimetil-4,4'-bipiridilo) es un químico tóxico utilizado mundialmente como herbicida. La etiología de la intoxicación por paraquat se debe en su mayoría a la ingestión suicida. Fisiopatológicamente, el pulmón, el hígado y el riñón son los órganos especialmente afectados por el paraquat debido a su alta saturación de oxígeno. La letalidad por intoxicación por paraquat es muy alta, cerca del $80 \%$, siendo la fibrosis pulmonar la causa más frecuente de muerte.

Objetivos: El siguiente documento tiene la intención de identificar los principales signos y síntomas que constituyen la presentación clínica de la intoxicación por paraquat en un paciente pediátrico. A pesar de la limitada bibliografía, se pretendió hacer una comparación de la presentación y el progreso clínico con otros casos de intoxicación por paraquat.

Material y métodos: Se expone el caso clínico de una

Dra. Cristina Contreras. Hospital Infantil Robert Reid Cabral, República Dominicana. crismi117@hotmail.com

Dra. Lisandra. Hospital Infantil Robert Reid Cabral, República Dominicana. Email Corona coronaq@gmail.com

Dra. Norys Domínguez. Hospital Infantil Robert Reid Cabral, República Dominicana. Email anyolinadominguez@hotmail. com

Dr. Miguel Mejía Sang miguel.mejia.sang@gmail.com joven de 15 ańos, la cual ingirió aproximadamente 250 $\mathrm{ml}$ de paraquat con intención autolítica. La misma presentó inicialmente afección en el tracto gastrointestinal, seguido de alteración de las pruebas hepáticas, renales y pancreáticas, y dificultad respiratoria.

Conclusiones: luego de describir la presentación clínica de nuestra paciente, y de compararla con la de otros casos similares, concluimos que el diagnóstico precoz y exhaustivo de la intoxicación por paraquat reduce significativamente su mortalidad debido a la actual inexistencia de un antídoto específico para dicho envenenamiento. Asimismo, recomendamos tener siempre presente la intoxicación por paraquat al momento de recibir pacientes con la presentación característica luego de la ingesta de sustancias desconocidas.

Palabras clave: Paraquat; herbicida; intoxicación; fibrosis pulmonar; mortalidad.

\section{Abstract}

Introduction: Paraquat (1, 1'-dimethyl-4, 4'-bipyridinium dichloride) is a toxic chemical used worldwide as an herbicide. Paraquat poisoning in its vast majority is due to a suicidal ingestion. Pathophysiologically, the lung, the liver and the kidney are the specially affected organs due to their high oxygen saturation. Paraquat poisoning has a very high mortality rate, close to $80 \%$, being pulmonary fibrosis the most common cause of death. 
Objetive: The following document has the intention to identify the principal signs and symptoms that constitute the clinical presentation of paraquat poisoning in a pediatric patient. Even though there is limited bibliography, we try to compare the clinical presentation and progress with other cases of paraquat poisoning.

Material and Methods: We present the case of a 15 years old woman, who ingested approximately $250 \mathrm{ml}$ of paraquat as a suicidal attempt. Even though there is limited bibliography, we try to compare the clinical presentation and progress with other cases of paraquat poisoning. She presented with lesions in the GI tract, followed by hepatic, renal and pancreatic alterations, and respiratory difficulty.

Conclusion: After describing the clinical presentation of our patient, and comparing it with those from other similar cases, we conclude that early diagnosis and a comprehensive, exhaustive management reduces mortality due to the inexistence of a specific antidote for this poisoning. As a result, we recommend that paraquat intoxication should always be kept in mind when receiving patient with the characteristic presentation after the intake of unknown substances.

Keyword: Paraquat; herbicida; intoxicación; pulmonar fibrosis; mortality

\section{Introducción}

El paraquat (dicloruro de 1,1'-dimetil-4,4'-bipiridilo) es un químico tóxico utilizado mundialmente como herbicida ${ }^{1}$. Ha sido clasificado como clase II o moderadamente peligroso tras su ingestión ${ }^{2}$.

La etiología de la intoxicación por paraquat se debe en su mayoría a la ingestión autolítica, sin embargo, existen casos de accidentes laborales, e ingestión inadvertida por niños o adultos en estado de ebriedad $^{1,3}$.

Sus propiedades herbicidas se deben a su capacidad de inhibir la reducción de NADP a NADPH, produciendo radicales libres que al reaccionar con el oxígeno forman radicales iónicos de superóxido y peróxido de hidrógeno produciendo la peroxidación lipídica de las membranas celulares (perdiendo su permeabilidad, cesando el transporte a través de la membrana), disfunción mitocondrial, necrosis, apoptosis, y la subsiguiente muerte celular, produciendo secundariamente una respuesta inflamatoria ${ }^{2}$.

Fisiopatológicamente, el pulmón, el hígado y el riñón son los órganos especialmente afectados por el paraquat debido a su alta saturación de oxígeno ${ }^{2,4}$.

Generalmente la intoxicación por paraquat ocurre por vía digestiva, sin embargo, se han reportado casos de intoxicación por vía inhalatoria, o vía dérmica si presenta pérdida de la integridad de la piel, aunque normalmente estas exposiciones producen lesiones localizadas y limitadas ${ }^{3,5}$.

La agresividad del cuadro depende de la cantidad ingerida del tóxico, por lo que se han descrito tres formas clínicas en función de la severidad del cuadro: intoxicación leve $(<20 \mathrm{mg} / \mathrm{kg}$, presentando una clínica aminorada, donde el daño hepatorrenal es benigno, existen síntomas gastrointestinales como dolor orofaríngeo, epigástrico, retroesternal y abdominal difuso, y alteración de las pruebas funcionales pulmonares, pero existe menos probabilidad de fibrosis), intoxicación moderada-severa (20-50 mg/kg, es la intoxicación más frecuente, presentando manifestaciones iniciales gastrointestinales, seguida de fallo hepatorrenal entre los días 2 y 5 , seguido de distrés respiratorio alrededor del día 7, con patrón radiológico alveolar, con evolución rápida a fibrosis pulmonar, que conduce a la muerte en tres 2-4 semanas), e intoxicación fulminante $(>50 \mathrm{mg} / \mathrm{kg}$, presentando ulceraciones orofaríngeas y perforación esofágica con mediastinitis, fallo cardíaco con miocarditis tóxica que resulta en insuficiencia cardíaca y arritmias graves, daño renal, hepático, respiratorio, pancreático, adrenal y neurológico, conllevando a la muerte en 24-48 horas por fallo multiorgánico) $)^{4,5}$.

La intoxicación por paraquat ocurre en 3 fases: fase gastrointestinal (durante las primeras 24 horas, y consta de náuseas, vómitos, dolor abdominal, disfagia, disfonía, sialorrea, diarrea y 
hemorragia digestiva, siendo la perforación esofágica o gástrica la principal complicación, que puede ser seguida por enfisema subcutáneo), fase hepatorrenal (ocurre a las 24-48 horas caracterizada por necrosis lobulillar hepática con aumento de la bilirrubina y transaminasas, y daño renal manifestado por aumento del nitrógeno ureico, creatinina y proteinuria), y fase pulmonar (evidenciada por acumulación alveolar de sangre, líquido y leucocitos, seguido por una proliferación acelerada de fibroblastos, resultando en fibrosis pulmonar irreversible) ${ }^{3,4,6}$.

El diagnóstico se realiza midiendo la presencia del tóxico cuantitativamente en jugo gástrico, orina y sangre mediante radioinmunoensayo, o cualitativamente mediante el test de ditionita ${ }^{3,5}$.

No existe antídoto para la intoxicación por paraquat, por lo que ante la sospecha deben iniciarse tempranamente medidas para reducir la absorción, aumentar la excreción y disminuir los efectos tóxicos sobre los órganos diana 5 .

El pronóstico de estos pacientes se encuentra relacionado principalmente de la concentración plasmática del agente y el tiempo transcurrido desde la ingesta. Si el paciente presenta datos de fallo multiorgánico desde el inicio, existe gran probabilidad de fallecer en las primeras 24-48 horas ${ }^{5,6}$.

La intoxicación por paraquat presenta una mortalidad mayor al $80 \%$, donde los pacientes afectados en su mayoría mueren en las dos semanas subsiguientes 5 .

\section{Reporte del caso}

Paciente femenina de 15 años de edad, sin antecedentes mórbidos conocidos, quien es llevada a sala emergencias tras haber ingerido aproximadamente $250 \mathrm{ml}$ de paraquat con intención suicida posterior a discusión con su madre. Fue tratada en un centro de su comunidad donde le fue realizado un lavado gástrico, y dada de alta con dieta suave que constase de Ensure $^{\oplus}$ (suplemento nutricional).

Tras presentar dificultad respiratoria resistente al tratamiento, fue traída a nuestro centro aproximadamente 96 horas después del incidente, presentando, además, murmullo vesicular disminuido, vómitos de contenido alimentario, sialorrea, disfonía, odinofagia, lengua eritematosa con aplanamiento de papilas gustativas y exudado blanquecino en cara dorsal, orofaringe eritematosa, dolor a la palpación en región anterior del cuello, secreciones vaginales blanquecinas y deshidratación moderada. Se realizó una radiografía de tórax que mostró aumento de la trama broncoalveolar bilateral, sin hallazgos en el parénquima, y ángulos costofrénico y cardiofrénico conservados.

Los laboratorios exhibieron aumento de los azoados (creatinina $7.9 \mathrm{mg} / \mathrm{dL}$, urea $115 \mathrm{mg} / \mathrm{dL}$, BUN $53.59 \mathrm{mg} / \mathrm{dL}$ ) y transaminasas (ALT 651 U/L, AST $208 \mathrm{U} / \mathrm{L}$ ), y $\beta$-HCG en sangre negativa. Tratada inicialmente con ceftriaxona, clindamicina, fluconazol, omeprazol, metilprednisolona, vitamina $\mathrm{E}$, vitamina $\mathrm{C}$, colutorios orales, hemodiafiltración veno-venosa continua (HDFVVC) y balance hídrico estricto.

A pesar del manejo, durante los siguientes días, las transaminasas y azoados continuaron elevándose, y la paciente comenzó a presentar oliguria, dolor de pecho en sedestación, y estertores roncus y crepitantes a la auscultación.

Durante la segunda semana de instauración del cuadro clínico, se encontró además náuseas, dolor epigástrico leve, escleras ictéricas y persistencia de oliguria.

Las pruebas analíticas lanzaron aumento de amilasa directa $504 \mathrm{U} / \mathrm{L}$, lipasa $287 \mathrm{U} / \mathrm{L}$, bilirrubina directa $3.94 \mathrm{mg} / \mathrm{dL}$, bilirrubina total $5.05 \mathrm{mg} / \mathrm{dL}$, fosfatasa alcalina $331 \mathrm{U} / \mathrm{L}, \mathrm{LDH} 686 \mathrm{U} / \mathrm{L}, \mathrm{GGT} 1,170$ $\mathrm{U} / \mathrm{L}$, leucocitos $20.4 \times 10^{3} / \mathrm{mm}^{3}$, y granulocitos $85.7 \%$, y disminución del calcio sérico $7.3 \mathrm{mg} /$ 
dL. Asimismo, hubo persistencia en la elevación de las azoados y transaminasas, por lo que se decide descartar etiología viral, arrojando HIV, HBsAG y HVC negativos.

Ante la evidente injuria hepática, renal y pancreática, se decide realizar sonografía abdominal, la cual presenta sólo hallazgos a favor de cambios parenquimatosos renales bilaterales.

Los resultados del cultivo de secreción vaginal arrojaron datos de Staphylococcus aureus, por lo que se sustituye la ceftriaxona y la clindamicina por levofloxacina.

Se agrega Ursacol $^{\circledast}$ (ácido ursodeoxicólico), ADEKForte $^{\circledast}$ (multivitamínico), y carbonato de calcio.

$\mathrm{Al}$ inicio de la tercera semana, la paciente experimentó mejoría de su cuadro clínico, diuresis adecuada, disminución de los azoados (creatinina 1.5 $\mathrm{mg} / \mathrm{dL}$, urea $70 \mathrm{mg} / \mathrm{dL}, \mathrm{BUN} 32.94 \mathrm{mg} / \mathrm{dL}$ ), por lo que se decide descontinuar hemodiálisis y espaciar terapia esteroidea.

Los días 18 y 19 evidenciaron recurrencia de ideas suicidas, apatía, y rechazo a la medicación, colaboración y alimentación, por lo que se refiere paciente y madre a departamento de Psicología. Asimismo, los días subsiguientes, la paciente refiere cefaleas leves, dolor torácico, y vértigos posicionales en normotensión, además presentó disnea, taquipnea, y saturación de oxígeno en $80 \%$. Se realiza radiografía de tórax, que muestra patrón intersticial, con ángulos conservados. Ante el nuevo cuadro, se adiciona ketorolaco para calmar dolor y, ante sospecha de fibrosis y lesión pulmonar, se inicia n-acetilcisteína, colchicina, y nebulización con budesonida.

Durante la cuarta semana, paciente exhibe exacerbación de dificultad respiratoria, retracciones costales y subcostales, aleteo nasal, $\mathrm{SaO} 272 \%$, llenado capilar ungueal en 3 segundos y cianosis de extremidades superiores, con palidez de piel y mucosas. Se decide agregar vancomicina y cefotaxima, ante sospecha de neumonía de origen a investigar.

Conjuntamente, la paciente presenta una disminución de los azoados (creatinina $0.9 \mathrm{mg} / \mathrm{dL}$, urea 37 $\mathrm{mg} / \mathrm{dL}, \mathrm{BUN} 17.19 \mathrm{mg} / \mathrm{dL}$ ), transaminasas (ALT $128 \mathrm{U} / \mathrm{L}$, AST $34 \mathrm{U} / \mathrm{L}$ ), bilirrubina directa 0.73 $\mathrm{mg} / \mathrm{dL}$, y bilirrubina total $1.41 \mathrm{mg} / \mathrm{dL}$.

El seguimiento de este caso clínico fue realizado por un equipo multidisciplinario de pediatras generales, neumólogos, gastroenterólogos, nefrólogos, infectólogos y psiquiatras. Durante el mismo, se pudo evidenciar las diversas fases en que ocurre la intoxicación por paraquat. Actualmente, la paciente se encuentra en estricta vigilancia, en espera de una positiva reacción al manejo interdisciplinario.

\section{Discusión}

El auto-envenenamiento con herbicidas es un problema mayor de salud pública en países en vías de desarrollo, con un estimado de 300000 muertes cada año solamente en la región Asia-Pacifico. Mientras que el envenenamiento por organofosforado constituye la mayoría de las admisiones hospitalarias, la mayoría de las fatalidades $(>50 \%)$ por paraquat constituyen la principal y única causa de muerte por envenenamiento con pesticidas en muchos países.

Existen pocos reportes de intoxicación pediátrica por ingesta de paraquat, pero a pesar de la limitada bibliografía, es posible hacer la comparación con distintos casos de intoxicación en pacientes adultos.

Janeela et al. (2017) expusieron el caso de un masculino de 28 años de edad, el cual consumió 10 $\mathrm{ml}$ de paraquat al $24 \%$ posterior a una disputa familiar. Fue encontrado inconsciente por su familia y fue llevado a sala de emergencias donde recibió un lavado gástrico, carbón activado, antieméticos, bloqueadores $\mathrm{H} 2$ y fluidos endovenosos como manejo inicial. A la evaluación se encontró oliguria, aumento progresivo de la urea $(97 \mathrm{mg} / \mathrm{dL})$ y 
creatinina, hipokalemia, además de presentar vómitos en múltiples ocasiones, ardor en la lengua, úlceras orales, dificultad para abrir la boca y para tragar alimentos sólidos. El paciente fue dado de alta en condiciones estables, con recomendaciones de realizar radiografía de tórax seriadas y espirometría para descartar fibrosis pulmonar.

En el caso expuesto por Kumar et al. (2016), un masculino de 50 años inhaló accidentalmente una sustancia desconocida (posteriormente identificada como paraquat) mientras fumigaba. Sus síntomas iniciales fueron nausea y vómitos, malestar oral, irritación nasal y ocular, y disnea. No presentó dificultad respiratoria. El hemograma, función renal, enzimas hepáticas, electrolitos y gases arteriales se encontraron dentro de los parámetros normales. De manera inicial fue manejado con un lavado gástrico, carbón y solución endovenosa. Luego fue trasladado a la UCI donde fue tratado con esteroides (metilprednisolona). Posteriormente fue diagnosticado con síndrome de disfunción multiorgánica. A pesar del esfuerzo realizado, el paciente expiró al sexto día ${ }^{8}$.

Yu et al. (2014) presentaron una femenina 42 ańos, la cual ingirió $>100 \mathrm{ml}$ de paraquat en un intento autolítico. Inicialmente presentó náuseas, vómitos e incomodidad justo después de la ingesta de la sustancia. Al momento de su admisión al centro recibió un lavado gástrico, además de infusiones de kaolin, manitol, metilprednisolona. Luego se le realizó hemoperfusión por 2 horas. A la examinación física, los signos vitales se encontraron dentro de los parámetros normales. En la cavidad oral se encontraron múltiples erosiones dolorosas. Después de dos meses, luego de presentar marcada mejoría y de haber descontinuado el esquema de tratamiento, el daño pulmonar agravó, acompañado de disfunción hepática, los cuales posterior a la reanudación del tratamiento con esteroides, recobraron su función normal. La paciente continúa en seguimiento?
Raghu et al. (2013) expusieron el caso de una femenina de 18 años, la cual consumió una cantidad desconocida de paraquat (en forma líquida) en su residencia. Los síntomas iniciales fueron dificultad para abrir la boca, disminución de la producción de orina, edema a nivel del cuello, erosión en mucosa oral (lengua, paladar y labios), sangrado oral y dificultad respiratoria. Además, presentó elevación de la urea $(221 \mathrm{mg} / \mathrm{dL})$ y la creatinina $(8,78 \mathrm{mg} / \mathrm{dL})$ sérica. Fue manejada con soluciones $\mathrm{EV}$, antieméticos, bloqueadores $\mathrm{H} 2$, hemodiálisis e intubación (por presentar edema laríngeo). La paciente expiró al décimo segundo día como consecuencia de fallo respiratorio ${ }^{10}$.

Con relación al caso clínico reportado, la paciente manifestó inicialmente un cuadro gastrointestinal florido sumado a una dificultad respiratoria, y seguido de aumento en las transaminasas y azoados. Fue manejado inicialmente con lavado gástrico y dieta suave, hasta que fue referido a nuestro hospital cerca de 96 horas luego del incidente e iniciándose el manejo con ceftriaxona, clindamicina, fluconazol, omeprazol, metilprednisolona, vitaminas $\mathrm{C}$ y $\mathrm{E}$, colutorios orales, hemodiafiltración veno-venosa continua (HDFVVC) y balance hídrico estricto. Esto resalta la importancia de un tratamiento oportuno que permita una estabilización temprana del cuadro clínico y de los parámetros de laboratorio, disminuyendo así las consecuencias a largo plazo y las fatalidades.

\section{Conclusión}

El diagnóstico precoz y el manejo exhaustivo de la intoxicación por paraquat reduce su mortalidad, ya que pequeñas cantidades de esta sustancia pueden llevar a consecuencias fatales. No existe un antídoto específico para este envenenamiento, por lo que su tratamiento sigue siendo de soporte en naturaleza, causando entonces una alta mortalidad. Es por esto que siempre se debe tener presente la intoxicación por paraquat al momento de recibir 
pacientes con daños gastrointestinales, respiratorios, renales y hepáticos luego de la ingesta de sustancias desconocidas.

\section{Bibliografía}

1. Centers for Disease Control and Prevention (CDC) [Internet]. Georgia: OPHPR; c2013 [Citado 2018 April 30]. Emergency Preparedness and response: Paraquat [aprox. 5 pantallas]. Disponible en: https://emergency. cdc.gov/agent/paraquat/basics/facts.asp

2. World Health Organization. The WHO Recommended Classification of Pesticides by Hazard and Guidelines to Classification. Rev IPCS. 2009. 3 (1); p 33, 36, 77.

3. Viales López, Glenda. Intoxicación por Paraquat. Med. leg. Costa Rica. 2014 Dec [Citado 2018 May 05]; 31 (2): 88-94.

4. Marín-Cuartas M, Berrouet-Mejía MC. Intoxicación por paraquat. Rev CES Med 2016. 30(1): 114-121.

5. Souto J, Palomar M. Intoxicación por paraquat. Rev TC. 2011; P 295-302.
6. Roberts DM, Buckley NA. Paraquat poisoning. Post TW, ed. UpToDate [Internet]. Waltham, MA: UpToDate Inc. 2016. (Citado 2018 May 02) [Aprox. 8 pantallas]. Disponible en: https://www.uptodate.com/contents/ paraquat-poisoning

7. Janeela MA, Oommen A, Misra AK, Ramya I. Paraquat poisoning: Case report of a survivor. Journal of Family Medicine and Primary Care. 2017;6(3):672-673.

8. Kumar H, Singh VB, Meena BL, Gaur S, Singla R. Paraquat Poisoning: A Case Report. Journal of Clinical and Diagnostic Research: JCDR. 2016;10(2): OD10-OD11.

9. Yu G, Kan B, Jian X, Wang J, Sun J, Song C. A case report of acute severe paraquat poisoning and long-term follow-up. Experimental and Therapeutic Medicine. 2014;8(1):233-236.

10. Raghu K, Mahesh V, Sasidhar P, Reddy PR, Venkataramaniah V, Agrawal A. Paraquat poisoning: A case report and review of literature. Journal of Family or Community Medicine. 2013;20(3):198-200. 